\title{
2. Normen und Standards in der KI
}

\author{
Axel Mangelsdorf
}

Es gibt immer mehr Anwendungen für KI - von optischer Erkennung, Datenanalyse und -manipulation über Chat-Bots bis zu virtueller Realität. Mit der steigenden Anzahl von Kl-Anwendungen in Unternehmen und dem immer größeren Nutzen für Verbraucher entsteht gleichzeitig eine Anzahl von neuen Herausforderungen. Dazu zählen Fragen der Interoperabilität der Systeme, Best Practices für den Einsatz von KI sowie Vertrauen und Sicherheit. Normen und Standards können einen wichtigen Beitrag dabei leisten, die wirtschaftliche Entwicklung der KI zu fördern und zugleich Vertrauen und Akzeptanz bei Mitarbeitern und Kunden zu stärken.

Für die KI-Anwendung sind Sicherheits- und Qualitätsnormen unerlässlich, da sie das Vertrauen in neue Technologien stärken, Kaufentscheidungen vereinfachen und damit die Markteinführung beschleunigen (Blind 2009). Normen funktionieren darüber hinaus als Katalysator für Innovationen, indem sie die Verbreitung von technologischem Wissen fördern, zur Erschließung neuer Märkte beitragen oder diese gar erst entstehen lassen. Die Entstehung von Normen und Standards ist jedoch ein komplexer Prozess, in den sich Unternehmen und auch andere interessierte Kreise wie Verbraucherorganisationen aktiv in technischen Komitees von Normungsorganisationen oder der Konsortialstandardisierung einbringen müssen. Für den Bereich der KI haben nationale und internationale Normungsorganisationen sowie informelle Standardisierungskonsortien den Bedarf nach Normen und Standards bereits erkannt, die KI-Normung steht jedoch noch am Anfang.

Dieser Beitrag gibt Auskunft, welche Normungsorganisationen, Standardisierungskonsortien und technische Komitees sich zurzeit aktiv mit dem Thema KI beschäftigen und welche Normen und Standards dort gesetzt werden. Zudem wird gezeigt, welche Normen und Standards es im Bereich KI aktuell gibt und welche Probleme diese Standards lösen. Schließlich werden mögliche künftige Handlungsräume erörtert und analysiert, welche Folgen die KI künftig für die Normungsarbeit selbst haben kann.

\section{KI-Normen und Normungsinitiativen}

Zwischen formellen Normungsorganisationen und informellen Standardisierungskonsortien gilt es zu unterscheiden: Formelle Normungsorganisationen entwickeln in 
offenen, transparenten und auf Konsens angelegten Prozessen formelle Normen oder de jure-Standards, während informelle Standardisierungskonsortien de factoStandards entwickeln. De facto-Standards entstehen durch Marktprozesse oder als Ergebnisse der konsortialen Standardisierung (Blind und Brock 2018). Formelle Normen haben im Vergleich zu informellen Standards eine oft höhere Qualität und besonders im Europäischen Binnenmarkt eine höhere Legitimität (Belleflamme 2002, Leiponen 2008). Formelle Normungsorganisationen sind zum Beispiel das Deutsche Institut für Normung (DIN) und die International Organization for Standardization (ISO). Zum Bereich der konsortialen Standardisierung zählen das amerikanische Institute of Electrical and Electronics Engineers Standards Association (IEEE-SA) oder der Zusammenschluss der großen US-amerikanischen IT-Unternehmen unter der Initiative Partnership on Artificial Intelligence to Benefit People and Society (Partnership on Al).

Normen lassen sich als Innovationsindikatoren heranziehen (Grupp et al. 2002). Neben den oben genannten Aspekten sind vor allem Interoperabilitäts-Normen in Industrien wichtig, die durch kurze Innovationszyklen gekennzeichnet sind. In diesen Industrien können Normen die Marktentwicklung von Produkten fördern oder gar erst entstehen lassen. Deshalb sind Normen nicht nur als nachgelagerte Indikatoren zu betrachten, sondern nehmen eher eine Katalysatorfunktion im Innovationsprozess ein (Blind 2009).

Mit Hilfe einer Datenbankabfrage in der Normendatenbank PERINORM ${ }^{7}$ lassen sich $\mathrm{KI}$-Normen recherchieren. So lassen sich etwa alle Normen finden, die die Begriffe "Artificial Intelligence" entweder im Titel oder im Abstract der Norm aufführen. Die Ergebnisse einer solchen Recherche zeigen, dass bis zum Jahr 2016 vor allem internationale Normen mit terminologischem Charakter entwickelt und in nationale Normenwerke übernommen, $d$. h. international verbreitet wurden.

Eine Norm für die Definition von KI-Terminologien ist die "ISO/IEC 2382:2015: Information technology - Vocabulary". Entwickelt hat sie das gemeinsame ISO/IEC technische Komitee "Informationstechnologie". Die Terminologienorm fasst IT-bezogene Definitionen zusammen, die zuvor in mehr als 30 Normen verteilt waren. Die Norm ISO/IEC 2382:2015 wurde von den formellen Normungsorganisationen in Tschechien, Deutschland, Frankreich, Japan, Litauen, Polen, der Türkei und den USA in nationale Normenwerke übernommen. Im Deutschen Institut für Normung wurde im Januar 2018 ein nationales Spiegelgremium zum ISO Subkomitee ISO/IEC JTC 1/ SC 42 gebildet. Der Normenausschuss NA 043-01-42 AA "Künstliche Intelligenz" wird aus dem Normenausschuss Informationstechnik und Anwendungen (NIA) her-

7 Siehe www.perinorm.com, zuletzt geprüft am 26.06.2018 
aus gegründet mit dem Ziel, deutsche Interessen auf der ISO Ebene angemessen zu berücksichtigen. Im Bereich der elektrotechnischen Normung, die in Deutschland durch die Deutsche Kommission Elektrotechnik Elektronik Informationstechnik in DIN und VDE (DKE) getragen wird, wurde mit der VDI-Richtlinie: VDINDE 3550 Blatt 1 „Computational Intelligence - Künstliche Neuronale Netze in der Automatisierungstechnik" eine einheitliche begriffliche Basis für die Verwendung der maßgeblichen Begriffe im industriellen Einsatz entwickelt. AK 801.0.8 befasst sich mit der Spezifikation und dem Entwurf autonomer/kognitiver Systeme. Der Verband und Deutsche Kommission Elektrotechnik Elektronik Informationstechnik (VDE/DKE) ist zusammen mit neun weiteren nationalen und internationalen Normungsorganisationen aus Österreich, Großbritannien, China, U.S.A., Irland und der Türkei Gründungsmitglied des weltweiten "Forums Open Community for Ethics in Autonomous and Intelligent Systems" (kurz: OCEANIS), das sich mit ethischen Fragen rund um autonome und intelligente Systeme auseinandersetzt. OCEANIS beabsichtig, Informationen austauschen und sich zu ihren jeweiligen Initiativen und Programmen hinsichtlich ethischer Standardentwicklung abzustimmen, gemeinsame Veranstaltungen zu organisieren und die Möglichkeit für abgestimmte Aktivitäten aufzuzeigen.

Neben der genannten Terminologienorm ISO/IEC 2382:2015 berühren weitere ISO/IEC-Aktivitäten das Thema KI. Das Subkomitee 7 (SC 7) des „ISO JTC 1 Software and systems engineering" produziert zum Beispiel horizontale Softwarenormen für Prozessmodelle und Best Practices. Das "SO Technische Komitee 299 Robotics" entwickelt u. a. Sicherheitsnormen für industrielle (ISO 10218:2012) und nichtindustrielle (ISO 13482:2014) Roboter. Im Europäischen Binnenmarkt hat die Norm EN ISO 13482:2014 im Zusammenspiel mit dem New Legislative Framework einen gesetzlichen Charakter, da sie als Harmonisierte Norm im Amtsblatt der Europäischen Union veröffentlicht wurde. Mit einer Einhaltung der Anforderungen der EN ISO 13482:2014 tritt die sogenannte Vermutungswirkung in Kraft. Das bedeutet, dass mit der Anwendung der Norm gleichzeitig die Anforderungen der EU-Maschinenrichtlinie (2006/42/EG) erfüllt werden und Hersteller oder Importeure das Produkt auf den Markt bringen dürfen (Egan 2002). Bei ISO wird das Thema KI seit 2017 in einem neuen Subkomitee ISO/IEC JTC 1/SC 42 Artificial Intelligence bearbeitet. Das ISO Subkomitee beschäftigt sich mit Normung im Bereich der KI und berät das JTC 1 , IEC und ISO zum Thema Entwicklung von KI-Anwendungen. Dem Subkomitee gehören Vertreter von nationalen Normungsorganisationen aus Österreich, Kanada, Finnland, Deutschland, Irland, Italien, der Schweiz und den USA an. Nicht stimmberechtigte Mitglieder (Observing Members) sind Dänemark und Schweden. Den Sekretariatsvorsitz des Subkomitees hat ein Vertreter des American National Standards Institute (ANSI) inne, den Stellvertretenden Vorsitz ein Vertreter des chinesischen Normungsinstituts. Bisher wurden von ISO/IEC JTC 1/SC 42 keine Normen veröffentlicht. 
Die ITU-T (ITU Telecommunication Standardization Sector) ist diejenige Abteilung innerhalb der ITU, die in technischen Komitee Standards - die bei ITU-T Recs bzW. Recommendations (Empfehlungen) genannt werden - auf dem Gebiet der Telekommunikation erarbeitet. ITU-T Recs sind freiwillig in der Anwendung, werden jedoch häufig in nationale Gesetzgebungen integriert. Die bisher veröffentlichten Empfehlungen behandeln inhaltlich beispielsweise die Internet Netzwerkarchitektur von Breitband DSL bis zu Next Generation Networks. Technische Arbeitsgruppen werden bei ITU Study Groups genannt und veröffentlichen Arbeitsprogramme, in denen die Themen der kommenden Jahre festgelegt werden. Die "Study Group 20 Internet of things (IoT) and smart cities and communities (SC\&C)" hat das Thema KI im Portfolio. Unter dem Titel "Artificial Intelligence and Internet of Things" wird derzeit in einem technischen Report analysiert, welche Interoperabilitätsfragen sich aus dem Zusammenspiel von KI und loT im Smart City Kontext ergeben und welche Standardisierungsaktivitäten ITU-T beginnen sollte.

\section{KI-Standards und Standardisierungsinitiativen}

Ein Blick auf KI-Innovationsindikatoren (KI entwickelnde Unternehmen, Patenanmeldungen) zeigt, dass amerikanische Unternehmen das Technologiefeld KI stark dominieren. Von den ca. 260 weltweit tätigen Unternehmen, die sich eindeutig mit der Entwicklung von KI beschäftigen, sind mehr als zwei Drittel in den USA tätig. Ebenso haben die meisten Anmelder von KI-Patenten ihren Wohnsitz in den USA (IPlytics 2018). Das amerikanische Standardisierungsinstitut IEEE-SA ist bei der Erstellung von $\mathrm{KI}-\mathrm{Standards}$ und bei KI-Standardinitiativen entsprechend ebenfalls Vorreiter.

Das Standardisierungsinstitut IEEE-SA hat mit der IEEE 1232-2010 "Artificial Intelligence Exchange and Service Tie to All Test Environments (AI-ESTATE)" einen Standard für die Fehler- und Systemdiagnose in Testumgebungen komplexer Systeme entwickelt. In komplexen Systemen wie z. B. im Flugzeugbau werden immer häufiger Techniken und Algorithmen der KI zur Fehler- und Systemdiagnose eingesetzt. Innerhalb dieser Algorithmen leiten Programme (sogenannte Inferenzmaschinen) Schlussfolgerungen über neues Wissen ab, die zur Lösung eines Problems führen. Der Standard IEEE 1232-2010 definiert Schnittstellen sowie Datenaustauschformate zwischen den Inferenzmaschinen, den Nutzern der Diagnosealgorithmen sowie angebundenen Datenbanken.

Die Norm IEEE 1232-2010 entwickelte die Institute of Electrical and Electronics Engineers Standards Association (IEEE-SA) im "IEEE Standards Coordinating Committee 20 (SCC 20) on Test and Diagnosis for Electronic Systems", und das Technische Komitee 91 "Electronics assembly technology" der Internationalen Elektrotechnischen Kommission überführte sie in eine internationale Norm. Diese wurde ins nationale Normenwerk von Großbritannien, den Niederlanden und Polen übernommen. 
Das Standardisierungsinstitut IEEE-SA hat im Jahr 2016 die IEEE-Initiative für ethische Betrachtungen in $\mathrm{KI}$ und autonomen System „IEEE Global Initiative for Ethical Considerations in Artificial Intelligence (Al) and Autonomous Systems (AS)" gegründet (IEEE-SA 2018). Die Initiative hat zum Ziel, das Vertrauen in KI zu erhöhen und schneller zu verbreiten. IEEE-SA veröffentlicht eine Guideline für Ethically Aligned Design, in der internationale Experten für KI, Robotik, Recht und Politik Empfehlungen für die Entwicklung von KI-Technologien geben. Zum Beispiel empfehlen sie, KI-Systeme so transparent zu gestalten, dass eine Evaluierung des Systems durch Dritte wie Regulierer oder Unfallbegutachter möglich ist. Damit soll sichergestellt werden, dass die Implementierung von Sicherheitsregeln und gesellschaftlichen Normen in KI-Systeme überprüft werden kann. In der IEEE Initiative gibt es bisher zehn spezifische Normenprojekte. Im Folgenden werden drei der zehn Projekte kurz vorgestellt.

Im Normenprojekt „Model Process for Addressing Ethical Concerns During System Design" wird eine Methodologie für KI-Softwareingenieure entwickelt, die sicherstellen soll, dass Anwender ethische Bedenken schon zu Beginn der Entwicklung bzw. des Softwarelebenszyklus analysieren und implementieren. Das IEEE Projekt "Transparency of Autonomous Systems" erarbeitet einen Guide für Entwickler von Autonomen Systemen, mit dessen Hilfe während des Entwicklungsprozesses die Transparenz des Systems bewertet werden kann. Transparenz bedeutet hier, dass die Benutzer von Pflegerobotern zum Beispiel jederzeit eine Begründung dafür verlangen können, warum das System diese oder jene Entscheidung getroffen hat. Transparenz bedeutet für das IEEE Projekt ebenfalls die Rückverfolgbarkeit von Unfällen. Das System muss interne Prozesse offenlegen, die zum Unfall geführt haben. Zum Beispiel sollen Entwickler analog zur Aufzeichnung von Daten in Flugschreibern (Black Box) gewährleisten, dass interne Daten und Sensordaten sicher gespeichert werden. Die Transparenz von Autonomen Systemen soll gesellschaftliches Vertrauen in disruptive Technologien wie fahrerlose Autos erhöhen. Das IEEE Projekt "Ontological Standard for Ethically Driven Robotics and Automation Systems" zielt darauf ab, einen ontologischen Standard zu entwickeln, mit dessen Hilfe die ethische und moralphilosophische Sprache und Theorie auf der einen Seite mit der ingenieurwissenschaftlichen Sprache auf der anderen Seite harmonisiert.

Neben dem Standardisierungsinstitut IEEE-SA haben die großen amerikanischen ITUnternehmen im Jahr 2016 ein Konsortium gegründet, das zum Ziel hat, für besseres Verständnis von KI in der Öffentlichkeit zu sorgen. Dem Konsortium „Partnership on Artificial Intelligence to Benefit People and Society" gehören die Unternehmen Amazon, DeepMind/Google, Facebook, IBM und Microsoft an. Etwa ein Jahr später trat auch Apple der Initiative bei. Deutsche Partner in der Gruppierung sind das Fraunhofer Institut für Arbeitswirtschaft und Organisation (IAO), SAP und Zalando. Das Kon- 
sortium will u.a. Industriestandards für KI-Anwendungen schaffen. Thematisch nennt die Gruppierung Bereiche wie Ethik, Transparenz, Sicherheit und Interoperabilität oder die Vertrauenswürdigkeit und Zuverlässigkeit der KI-Technologie (Partnership on Al 2018). Bisher wurden jedoch noch keine Industriestandards oder Best Practices veröffentlicht. Ebenfalls ist unklar, ob die Gruppierung Industriestandards über die Partnership on Al veröffentlicht oder ob die Unternehmen in technischen Gremien von ITU an internationalen Standards mitarbeiten. Schließlich sind Unternehmen wie Google auch in ITU Mitglieder.

\section{Handlungsräume}

Die Normung und Standardisierung für die KI steht noch am Anfang. Im Vergleich zu anderen Innovationsindikatoren (Anzahl der KI-Unternehmen, Patente, wissenschaftliche Veröffentlichungen) gibt es noch wenige Normen und Standards. Gleichzeitig steigen mit der Anzahl von Produkten und Anwendungen, die KI einsetzen, auch die Bedenken der Anwender und Verbraucher. Eine repräsentative Umfrage des Branchenverbandes Bitkom (Bitkom 2017) zeigt beispielsweise, dass eine Mehrheit der Bundesbürgerinnen und Bundesbürger befürchtet, dass der Einsatz von KI zu Machtmissbrauch und Manipulation führen kann. Die international am meisten verbreitete Norm ist eine Terminologienorm. Normen, die dem Nutzer von KI-Anwendungen Sicherheit garantieren, ethische Normen für KI-Algorithmen und Normen für die private Datensicherheit von KI-Anwendungen fehlen bisher weitgehend. Vor diesem Hintergrund lässt sich ein Normungs- und Standardisierungsbedarf für folgende Aspekte benennen.

1. Terminologie: Normen für die Definition von KI-Begriffen schaffen eine gemeinsame technische Sprache. Besonders internationale Normen verhindern nationale Insellösungen und reduzieren Handelshemmnisse.

2. Interoperabilität: Normen für Interoperabilität oder Schnittstellenstandards ermöglichen, dass verschiedene KI-Systeme zusammenarbeiten können und damit Netzwerkeffekte realisieren. Interoperabilität verringert Abhängigkeit von einzelnen Komponenten und Systemen und steigert die Produktvielfalt.

3. Sicherheit und Qualität: Normen für Sicherheit und Qualität erhöhen die Transparenz und Akzeptanz von KI-Technologien bei Verbrauchern. Dadurch werden Transaktionskosten gesenkt. Sicherheits- und Qualitätsstandards bilden oft die Grundlage für eine Zertifizierung durch Dritte. Im Zusammenspiel mit verpflichtenden Regulierungen können Sicherheits- und Qualitätsstandards de facto rechtsverbindlichen Charakter bekommen.

4. Ethische Standards: Nach Einschätzung von Bitkom (Bitkom 2017) und PwC (PwC 2017) ist die Formulierung von ethischen Standards fundamental für die Realisie- 
rung von $\mathrm{KI}$ als Wachstumstreiber. $\mathrm{KI}$ ist nicht nur eine technologische Weiterentwicklung, denn KI hat auch das Potenzial, das Selbstbild des Menschen gegenüber Maschinen, das soziale Gefüge und die politische Meinungsbildung radikal zu verändern. Ethische Standards müssen deshalb sicherstellen, dass KI-Systeme und Algorithmen menschengerecht gestaltet werden. Um Handelsbarrieren zu verhindern, sind internationale Lösungen anzustreben.

\section{Ausblick}

Derzeit lässt sich noch nicht mit Sicherheit abschätzen, wie Normung und Standardisierung der KI in den nächsten Jahren aussehen werden. Vorstellbar sind Szenarien, in denen einzelne führende KI-Unternehmen Industriestandards einführen (beispielsweise über die "Partnership on $\mathrm{Al}^{\prime}$ ), die sich am Markt durchsetzen. Da sich die KIIndustrie geographisch derzeit stark in den USA konzentriert, ist es auch ein wahrscheinliches Szenario, dass amerikanische Normungsorganisationen im Vorteil sind, da hier die relevanten Expertinnen und Experten in großer Zahl vorhanden sind, die sich in den Normungsgremien beteiligen und die Inhalte der Normen definieren. Dieses Szenario ist umso wahrscheinlicher, als die in den USA ansässige Normungsorganisation IEEE-SA einen Vorsprung beim Thema KI hat.

Normen und Standards haben nicht nur einen Einfluss auf die Entwicklung der $\mathrm{KI}$, sondern die Digitalisierung im Allgemeinen, und die KI im Speziellen hat umgekehrt ebenso einen zunehmenden Einfluss auf die Normungsarbeit. Digitale Techniken, wie Webkonferenzen, werden immer häufiger verwendet, um die Hürden gerade für kleine und mittlere Unternehmen, sich an der Normung zu beteiligen, zu senken und somit eine breitere Beteiligung aller relevanten Parteien sicherzustellen. Normen und Standarddokumente in elektronischer Form bzw. maschinenlesbare und verarbeitbare Normeninhalte erlauben eine schnellere Anwendung von Normen, eine einfachere Identifizierung von Schnittstellen und damit eine einfachere und effizientere Nutzung (Birner et al. 2017). Software-Algorithmen simulieren schon heute das Verhalten von technischen Komponenten, und die Ergebnisse solcher Simulationen gehen in die Normungsarbeit ein. Zukünftig können KI-unterstützte Algorithmen die Spezifikationen der Algorithmen selbst verändern, was den Normungsprozess beschleunigen könnte. Normungsorganisationen werden mit der Frage konfrontiert, welche Art von Algorithmen (Baysianisch, Regression etc.), Daten und Computer verwendet werden. Genieren KI-Algorithmen eigenständig neue Technologien, wird auch die Frage nach den intellektuellen Eigentumsrechten aufgeworfen. Da nur Menschen und keine Maschinen Besitzer von solchen Schutzrechten sein können, unterliegen KI-generierte Technologien auch keinem Patentschutz. Werden KI-generierte Technologien in die Normung eingebracht, lassen sich ohne Patentschutz auch keine Lizenzeinnahmen erzielen (Alderman und Newman 2018). 
Der Normungsprozess ist vereinfacht als ein Entscheidungsbaum zu verstehen. Die Normungsexperten wählen eine bestimmte Verzweigung und diskutieren anschlieBend, welche Verzweigung im Entscheidungsbaum sie als nächstes wählen. Diese Vorgehensweise ist zeitintensiv und benötigt Ingenieurswissen und -kapazitäten. Hypothetisch ist somit auch vorstellbar, dass die KI den Normungsprozess beschleunigt, indem Algorithmen die Entscheidungsfindung übernehmen. Unternehmen in der Normung könnten zukünftig ihre Parameter und Ziele in KI-unterstützte Algorithmen eingeben und den optimalen Pfad errechnen, Grafiken erstellen und den Text des Standards schreiben (Alderman und Newman 2018). Dies wäre allerdings ein Szenario, denn ohne die Mitwirkung von Menschen in den Normungsprozessen ginge den Unternehmen eine wichtige Quelle für den Austausch technologischen Wissens verloren. Denn besonders für kleine und mittlere Unternehmen (KMU) ist der Wissenstransfer durch die Beobachtung von Wettbewerbern und die nicht-dokumentierten Gespräche in den Gremien ein wichtiges Motiv, um an der Normung teilzunehmen (Blind und Mangelsdorf 2016). 


\section{Literatur}

Alderman, R.; Newman, D. (2018): Could Al Take Over Standard Development Organizations? Online verfügbar unter https://www.law360.com/articles/1016289, zuletzt geprüft am 09.03.2018.

Belleflamme, P. (2002): Coordination on formal vs. de facto standards: A dynamic approach. European Journal of Political Economy, Volume 18 (Issue 1,), 153-176.

Birner, N., Gieschen, J.-H., Kudernatsch, W., Moorfeld, R., Weiler, P.; Schotten, H. (2017): Die Rolle der Normung 2030 und Gestaltungsoptionen unter Berücksichtigung der technologiespezifischen Besonderheiten der IKT in der Normung und Standardisierung (Abschlussbericht). Studie im Auftrag des Bundesministeriums für Wirtschaft und Energie. Online verfügbar unter https://www.bmwi.de/Redaktion/DE/Publikationen/Studien/rolle-der-normung-2030.pdf?_blob=publicationFile\&v=16, zuletzt geprüft am 09.03.2018.

Bitkom (2017): Künstliche Intelligenz. Online verfügbar unter https://www.bitkom.org/Presse/ Anhaenge-an-PIs/2017/11-November/Bitkom-Charts-PK-Al-15-11-2017-final.pdf, zuletzt geprüft am 09.03.2018.

Blind, K. (2009): Normung als Katalysator für Innovationen, Inaugurationsrede, Normung als Katalysator für Innovationen, Inaugurationsrede, August 2009, DIN e.V., Berlin.

Blind, K.; Brock, M. (2018): Patentierung und Standardisierung: Leitfaden für modernes Innovationsmanagement. Leitfaden für modernes Innovationsmanagement: Beuth Verlag.

Blind, K.; Mangelsdorf, A. (2016): Motives to standardize: Empirical evidence from Germany. Technovation (February-March), 13-24.

Deutsche Kommission Elektrotechnik Elektronik Informationstechnik in DIN und VDE (DKE). AK 801.0.8 Spezifikation und Entwurf autonomer / kognitiver Systeme. Online verfügbar unter https://www.dke.de/de/ueber-uns/dke-organisation-auftrag/dke-fachbereiche/ dke-gremium?id=3006525\&type=dke\%7Cgremium, zuletzt geprüft am 25.07.2018.

Egan, M. (2002): Setting Standards: Strategic Advantages in International Trade. Business Strategy Review, 13 (1), 51-64.

Grupp, H., Dominguez Lacasa, I.; Friedrich-Nishio, M. (2002): Das deutsche Innovationssystem seit der Reichsgründung. Indikatoren einer nationalen Wissenschafts- und Technikgeschichte in unterschiedlichen Regierungs- und Gebietsstrukturen ; mit 11 Tabellen (Technik, Wirtschaft und Politik, Bd. 48). Heidelberg: Physica-Verl.

IEEE-SA. (2018): The IEEE Global Initiative on Ethics of Autonomous and Intelligent Systems. Zugriff am 09.03.2018. Online verfügbar unter https://standards.ieee.org/develop/ indconn/ec/autonomous_systems.html, zuletzt geprüft am 09.03.2018.

IPlytics. (2018): IPlytics Platform. Online verfügbar unter https://platform.iplytics.com, zuletzt geprüft am 09.03.2018.

Leiponen, A. E. (2008): Competing through cooperation: The organization of standard setting in wireless telecommunications. Management Science, Volume 54 (Issue 11), 1904-1919. 
Partnership on Al. (2018): Partnership on Artificial Intelligence to Benefit People and Society. Online verfügbar unter https://www.partnershiponai.org, zuletzt geprüft am 09.03.2018.

PricewaterhouseCoopers (2017): Sizing the prize What's the real value of Al for your business and how can you capitalise? Online verfügbar unter https://www.pwc.com/gx/en/issues/ analytics/assets/pwc-ai-analysis-sizing-the-prize-report.pdf, zuletzt geprüft am 09.03.2018.

\section{(c) (1)}

Dieses Kapitel wird unter der Creative Commons Namensnennung 4.0 International Lizenz http://creativecommons.org/licenses/by/4.0/deed.de) veröffentlicht, welche die Nutzung, Vervielfältigung, Bearbeitung, Verbreitung und Wiedergabe in jeglichem Medium und Format erlaubt, sofern Sie den/die ursprünglichen Autor(en) und die Quelle ordnungsgemäß nennen, einen Link zur Creative Commons Lizenz beifügen und angeben, ob Änderungen vorgenommen wurden.

Die in diesem Kapitel enthaltenen Bilder und sonstiges Drittmaterial unterliegen ebenfalls der genannten Creative Commons Lizenz, sofern sich aus der Abbildungslegende nichts anderes ergibt. Sofern das betreffende Material nicht unter der genannten Creative Commons Lizenz steht und die betreffende Handlung nicht nach gesetzlichen Vorschriften erlaubt ist, ist für die oben aufgeführten Weiterverwendungen des Materials die Einwilligung des jeweiligen Rechteinhabers einzuholen. 